\title{
Multivariate Classification of Brugada Syndrome Patients Based on the Autonomic Response During Sleep, Exercise and Head-up Tilt Testing
}

\author{
Mireia Calvo ${ }^{1}$, Virginie Le Rolle ${ }^{2}$, Daniel Romero ${ }^{1}$, Nathalie Béhar ${ }^{2}$, Pedro Gomis $^{3}$, Philippe Mabo $^{2}$, \\ Alfredo Hernández ${ }^{2}$ \\ ${ }^{1}$ Institute for Bioengineering of Catalonia (IBEC), Barcelona, Spain \\ ${ }^{2}$ Univ Rennes, CHU Rennes, Inserm, LTSI UMR 1099, Rennes, France \\ ${ }^{3}$ Universitat Politècnica de Catalunya, Barcelona, Spain
}

\begin{abstract}
Several autonomic markers were estimated overnight and during exercise and head-up tilt (HUT) testing for 44 $B S$ patients, to design classifiers capable of distinguishing patients at different levels of risk. The classification performance of predictive models built from the optimization of a step-based machine-learning method were compared, so as to identify those autonomic protocols and markers best distinguishing between symptomatic and asymptomatic patients. Although exercise and HUT testing together led to better predictive results than when they were separately assessed, among all analyzed combinations, the night-based classifier presented the best performance $(A U C=95 \%)$, using the least amount of features. This optimal features subset was mostly composed of markers extracted between 4 a.m. - 5 a.m. Thus, results provide further evidence for the role of nighttime analysis, mainly during the last hours of sleep, for risk stratification in BS.
\end{abstract}

\section{Introduction}

Brugada syndrome (BS) is a genetic arrhythmogenic disorder presenting an abnormal pattern on the electrocardiogram (ECG), characterized by a distinctive ST-segment elevation in right precordial leads; associated with a high risk for sudden cardiac death (SCD) due to ventricular fibrillation (VF) in absence of structural cardiopathies [1].

After diagnosis, implantable cardioverter defibrillators (ICD) are the only proven effective treatment thus far and current guidelines recommend an ICD implantation in symptomatic BS patients [1]. However, it is difficult to perform an appropriate BS patient selection for device implantation when no symptoms are documented, since i) this population shows a relative low risk of cardiac events (estimated at less than 1\% [2]) and ii) ICD implantation can be associated with complications related to surgery and inappropriate shocks. Thus, risk stratification to determine the best therapeutic strategy remains challenging for asymptomatic patients, who represent around $60 \%$ of diagnosed patients.

Major cardiac events in BS patients most commonly occur during periods of increased parasympathetic tone, at rest or during sleep [3], and studies based on positron emission tomography have demonstrated autonomic dysfunctions in this population [4]. Although previous studies based on long-term spontaneous measurements have led to inconclusive results [5-7], it has been hypothesized that the application of controlled autonomic maneuvers may improve reproducibility of the observed markers. Indeed, some studies have already reported the interest of autonomic evaluation during controlled exercise testing $[8,9]$, among other autonomic tests [10], for the prediction of cardiac events in BS.

In previous works, we reported significant differences in the autonomic function of symptomatic and asymptomatic BS patients overnight [7] and during exercise [11-13] and head-up tilt (HUT) testing $[14,15]$. In this paper, we further explore the potential of heart rate variability (HRV) markers for BS classification, by combining features extracted from these experimental conditions.

\section{Materials and methods}

\subsection{Study population}

The standard 12-lead ECG recordings from 44 patients suffering from BS were collected for 24 hours, including exercise and HUT tests. They were acquired during a prospective, multicenter study, led by the Cardiology department of the Rennes University Hospital (CHU de Rennes), in France. Participants were enrolled in 6 French hospitals located in Rennes, Saint Pierre de la Réunion, Nantes, Bordeaux, Brest and La Rochelle. The study protocol was approved by the respective local ethics committees and all patients provided their written informed con- 
sent to participate in the study.

According to current international guidelines [1], BS was diagnosed when a coved ST-segment elevation $(\geq 0.2$ $\mathrm{mV}$ ) was observed in at least one right precordial lead placed in the $2^{\text {nd }}, 3^{\text {rd }}$ or $4^{\text {th }}$ intercostal space. Moreover, patients were classified as symptomatic and asymptomatic, based on their medical history, so as to characterize populations with different levels of risk.

Participant ages ranged from 19 to 73 years old ( $45.45 \pm$ 12.78 years old) and $38(86.5 \%)$ were males. Among these 44 patients, eleven had experienced symptoms of ventricular origin (symptomatic group): syncope $(72.7 \%)$, cardiac arrest (18.2\%) and, less frequently, palpitations and nocturnal convulsions (9.1\%). All these patients had been implanted with an ICD. The rest of the population (33 patients) were labelled as asymptomatic. From these 33 patients, 10 had also been implanted (30.3\%), following clinical recommendations after a positive EPS (Electrophysiological Study) test [1].

\subsection{Study design}

ECG signals were acquired with a Holter monitor (ELA medical, Sorin Group, Le Plessis Robinsson, France), at a sampling frequency of $1000 \mathrm{~Hz}$. From the whole recording, autonomic analysis was focused on three specific periods: 1) during exercise, 2) HUT testing and 3) overnight.

\subsubsection{Exercise testing}

Following the American Heart Association recommendations [16], load was progressively augmented until the patient reached $80 \%$ of his/her theoretical maximum heart rate $(M H R=220-$ age [17]). The test was performed in a cyclo ergometer (Ergoline 900 Egamed, Piestany, Slovakia) and divided in the following phases:

- Exercise phase: initial load of $50 \mathrm{~W}$ (30 W for women), for 2 minutes (warm-up); followed by a load of $80 \mathrm{~W}$ (50 $\mathrm{W}$ for women) for 2 minutes and then incrementing $20 \mathrm{~W}$ every 2 minutes (incremental exercise).

- Recovery phase: fixed load of $50 \mathrm{~W}$ (30 W for women), for 3 minutes (active recovery); followed by a total cessation of effort for 3 minutes (passive recovery).

\subsubsection{Head-up tilt testing}

It was performed in the morning, in fasting conditions, according to the following protocol:

- Baseline: 10 minutes in supine position.

- Tilting phase: 45 minutes at $60^{\circ}$ of table (Sissel, Sautron, France) inclination. All positive cases were excluded from the study.

- Post-tilt resting phase: 10 minutes in supine position.

\subsubsection{Nighttime analysis}

From the recorded 24 hours, the period from midnight to 6 a.m. was identified. Then, nighttime analysis was focused on the analysis of 6 consecutive 1-hour segments.

\subsection{HRV features}

From the 12-lead ECG signals acquired during each autonomic test and during the night, RR-interval and R-peak amplitude series were extracted by using a noise-robust wavelet-based algorithm [18]. A cubic-spline interpolation was then applied to these series, in order to obtain uniformly sampled data at a rate of $4 \mathrm{~Hz}$.

Then, based on our previously reported analyses on the autonomic function of BS patients during A) exercise [11, 12], B) HUT testing [14] and C) sleep [7], several HRV markers were calculated for each patient, to build a set of candidate features to be used for classification purposes.

\subsubsection{Exercise testing}

Spectral non-stationary HRV markers were extracted by means of a Smoothed Pseudo Wigner Ville distribution (SPWVD) method, described in detail elsewhere [11], that adapts frequency bands to respiratory information.

These markers were obtained from the averaged values of $L F, L F_{n u}, H F, H F_{n u}$ and $L F / H F$ for nonoverlapped 1-minute windows, including the warm-up phase, the first 3 minutes and the last minute of exertion, as well as the active and passive recovery periods. Moreover, based on a previous study on heart rate complexity (HRC) captured by power-law scaling analysis where significant differences between symptomatic and asymptomatic BS patients were noted [12], the $\beta$ slopes measured during the whole exercise and recovery phases were calculated.

\subsubsection{Head-up tilt testing}

For the design of classifiers based on HUT testing data, the same HRV markers were extracted. In this case, the mean for the whole baseline period was subtracted from each estimated HRV time series and, in order to reduce classification computational cost, only the first 5 minutes after tilting and the time period between 11-15 minutes in upright position were included, based on a previous study [14], where the most significant autonomic differences between symptomatic and asymptomatic BS patients were found before the $15^{\text {th }}$ minute in upright position.

\subsubsection{Nighttime analysis}

According to a previously reported study on the autonomic function of BS patients at night [7], classical tempo- 
ral and spectral, as well as non-linear, HRV markers were calculated for each night hour.

\subsection{Classification methodology}

A 4-step machine-learning approach was applied to features extracted from each experimental condition and to all possible combinations of the available autonomic tests:

1. Feature extraction. The above-mentioned HRV features were extracted for each patient. A matrix of candidate features $R_{M}^{N}$, of size $N \times M$, with $N=44$ patients was estimated. Values of $M$ depended on the selected set of experimental conditions: exercise $(M=62)$; HUT ( $M=50)$; nighttime $(M=66)$; exercise and HUT ( $M=112)$; exercise and night $(M=128)$; HUT and night $(M=116)$; and exercise, HUT and night $(M=178)$.

2. Feature conditioning. To reduce the effect of markers measured at different scales and the impact of imbalanced groups of patients, a standardization followed by an ADASYN-based class balancing method [19] was applied to the ensemble of candidate features, leading to matrices $F_{M}^{N}$ and $F_{M}^{N_{b}}$, respectively, where $N_{b}>N$ refers to the number of observations after class balancing.

3. Feature selection. Patient subsets were selected for training ( $N_{t r}, 75 \%$ of samples) and testing ( $N_{t e}$, the rest). Then, the minimal feature dimension $M_{w}<M$, maximizing classification performance, was estimated using a twostep approach: 1) a filter method [20] discarding the $25 \%$ least relevant markers and, 2) a wrapper method [21], optimized based on the maximum $A U C$ (area under the ROC curve), selecting the final feature subset as those markers appearing more than a specific number of times $L$.

4. Classification. Classifiers based on Linear Discriminant Analysis (LDA) were then designed to distinguish between symptomatic and asymptomatic populations, based on features selected on the previous step. Finally, classification performance was evaluated by measuring the $A U C$ and applying a 10-times 4-fold cross-validation. Moreover, the capability of developed classifiers on detecting symptomatic and asymptomatic patients was quantified by the classical sensitivity $(S e)$ and specificity $(S p)$ measures.

\section{Results}

\subsection{Single-test analysis}

In a first phase, three single-test classifiers were separately built with features extracted from each experimental setup. Regarding exercise analysis, the best classifier was designed when only those markers appearing more than $L=18$ times after wrapper feature selection were kept. The final subset contained $M_{w}=31$ features and led to an $A U C=0.89 \pm 0.03$, with a sensitivity of $S e=0.88 \pm 0.04$ and a specificity of $S p=0.74 \pm 0.11$. Although this predictive model did not reach the classifier performance reported in our previous work $\left(A U C=92 \%\right.$ for $M_{w}=22$ features) [11], probably because it was based on a reduced amount of observations for model training, the proposed classification methodology demonstrated an acceptable generalization capability, since it was consistent with the previous study where the same optimal $L$ was selected.

The optimal $A U C$ for classifiers based on HUT testing data was found for $L=12$, leading to a final subset containing $M_{w}=31$ features with a performance of $A U C=$ $0.88 \pm 0.06, S e=0.74 \pm 0.08$ and $S p=0.86 \pm 0.15$. Similar results were thus observed for the classifiers based on exercise and HUT tests, with a slightly improved specificity at the expense of worsening sensitivity for the HUTbased classifier. Since our priority was to correctly identify symptomatic BS patients and HUT-based classification presented a lower sensitivity, exercise testing seemed to be more suitable for high-risk BS patients identification.

Finally, the optimal $A U C$ regarding nighttime analysis was found for $L=15$, leading to the best performance among single-test classifiers $(A U C=0.95 \pm 0.03, S e=$ $0.94 \pm 0.08, S p=0.80 \pm 0.12)$, using the least amount of HRV features $\left(M_{w}=26\right)$.

\subsection{Multi-test analysis}

Table 1 summarizes the optimal $A U C$ obtained for each single- and multi-test classifier, together with their associated final dimensions $\left(M_{w}\right)$.

Table 1. Mean and standard deviation of optimal $A U C$ values, and final dimensions, for all classifiers.

\begin{tabular}{lcr}
\hline \hline Classifier & $A U C$ & $M_{w}$ \\
\hline Exercise & $0.89 \pm 0.03$ & 31 \\
HUT & $0.88 \pm 0.06$ & 31 \\
Night & $0.95 \pm 0.03$ & 26 \\
Exercise - HUT & $0.91 \pm 0.04$ & 30 \\
Exercise - Night & $0.84 \pm 0.08$ & 26 \\
HUT - Night & $0.89 \pm 0.04$ & 23 \\
Exercise - HUT - Night & $0.92 \pm 0.04$ & 60 \\
\hline \hline
\end{tabular}

Based on the results, the predictive value of exercise testing $(A U C=0.89 \pm 0.03)$ increased by adding HUT testing information $(A U C=0.91 \pm 0.04)$, and it was even improved by including night data $(A U C=0.92 \pm 0.04)$. However, the best classification results were found when nighttime was separately analyzed $(A U C=0.95 \pm 0.03)$.

Among the 26 final features composing this optimal night-based classifier, only one HRC marker was kept, acquired between 4 a.m. - 5 a.m. The remaining parameters mostly belong to temporal HRV measures, although 6 spectral features were also retained. Regarding analyzed 
hours, parameters were obtained along the whole night; however, the time period including more features turned to be 4 a.m - 5 a.m.

\section{Conclusions}

These findings provide further evidence for the relevant role of autonomic response analysis during nighttime, mostly during the last hours of sleep, to identify BS patients at high risk. The results concur with previous studies reporting that most major cardiac events in BS occur at rest and during the last hours of sleep, when parasympathetic activity is predominant [3]. Moreover, as suggested by previous studies reporting alterations in the autonomic modulation and heart rate variability and complexity of BS patients at higher risk [5-7], the proposed autonomic markers seem to unmask pathophysiological differences on patients at high risk that may be useful for risk stratification. Since the proposed predictive model showed an improved performance with respect to previous works in the field, it is presented as a potential complementary instrument to electrophysiological markers, so as to better identify those asymptomatic patients at risk that may benefit from a defibrillator implantation.

\section{References}

[1] S. G. Priori, et al., "Task Force for the management of patients with ventricular arrhythmias and the prevention of sudden cardiac death of the European Society of Cardiology (ESC) endorsed by: Association for European Paediatric and Congenital cardiology (AEPC)," Europace, vol. 17, pp. 1601-1687, 2015.

[2] V. Probst, et al., "Long-term prognosis of patients diagnosed with brugada syndrome results from the FINGER Brugada syndrome registry," Circulation, vol. 121, no. 5, pp. 635-643, 2010.

[3] K. Matsuo, et al., "The circadian pattern of the development of ventricular fibrillation in patients with Brugada syndrome," Eur Heart J, vol. 20, no. 6, pp. 465-470, 1999.

[4] M. Paul, et al., "Autonomic dysfunction in patients with Brugada syndrome: further biochemical evidence of altered signaling pathways," Pacing Clin Electrophysiol, vol. 34, no. 9, pp. 1147-1153, 2011.

[5] K. Nakazawa, et al., "Autonomic imbalance as a property of symptomatic Brugada syndrome," Circ J, vol. 67, no. 6, pp. 511-514, 2003.

[6] T. Tokuyama, et al., "Deterioration of the circadian variation of heart rate variability in Brugada syndrome may contribute to the pathogenesis of ventricular fibrillation," J Cardiol, vol. 64, no. 2, pp. 133-138, 2014.

[7] M. Calvo, et al., "Heart rate differences between symptomatic and asymptomatic Brugada syndrome patients at night," Physiol Meas, vol. 39, no. 6, pp. 065002, 2018.

[8] H. Makimoto, et al., "Augmented ST-segment elevation during recovery from exercise predicts cardiac events in pa- tients with Brugada syndrome," J Am Coll Cardiol, vol. 56, no. 19 , pp. 1576-1584, 2010.

[9] M. Subramanian, et al., "The utility of exercise testing in risk stratification of asymptomatic patients with type 1 Brugada pattern," J Cardiovasc Electrophysiol, vol. 28, pp. 677-683, 2017.

[10] M. A. B. Bigi, et al., "Significance of cardiac autonomic neuropathy in risk stratification of Brugada syndrome," $E u$ ropace, vol. 10, no. 7, pp. 821-824, 2008.

[11] M. Calvo, et al., "Multivariate classification of Brugada syndrome patients based on autonomic response to exercise testing," PLoS ONE, vol. 13, no. 5, p. e0197367, 2018.

[12] M. Calvo, et al., "Heart rate complexity analysis in Brugada syndrome during physical stress testing," Physiol Meas, vol. 38, no. 2, pp. 387-396, 2017.

[13] M. Calvo, et al., "Recursive model identification for the analysis of the autonomic response to exercise testing in Brugada syndrome," Artif Intell Med, 2018.

[14] M. Calvo, et al., "Time-frequency analysis of the autonomic response to head-up tilt testing in Brugada syndrome," in Computing in Cardiology Conference (CinC), 2017, 2017.

[15] M. Calvo, et al., "Model-based analysis of the autonomic response to head-up tilt testing in Brugada syndrome," Comput Biol Med, vol. 103, pp. 82-92, 2018.

[16] R. J. Gibbons, et al., "ACC/AHA 2002 guideline update for exercise testing: summary article: a report of the American College of Cardiology/American Heart Association Task Force on practice guidelines (committee to update the 1997 exercise testing guidelines)," J Am Coll Cardiol, vol. 40, no. 8, pp. 1531-1540, 2002.

[17] S. Fox 3rd and W. Haskell, "Physical activity and the prevention of coronary heart disease," J Urban Health, vol. 44, no. 8, pp. 950-965, 1968.

[18] J. Dumont, et al., "Improving ECG beats delineation with an evolutionary optimization process," IEEE Trans Biomed Eng, vol. 57, no. 3, pp. 607-615, 2010.

[19] H. He, et al., "ADASYN: Adaptive synthetic sampling approach for imbalanced learning," in 2008 IEEE International Joint Conference on Neural Networks (IJCNN), 2008, pp. 1322-1328.

[20] M. Robnik-Šikonja and I. Kononenko, "Theoretical and empirical analysis of relieff and rrelieff," Mach Learn, vol. 53, no. 1-2, pp. 23-69, 2003.

[21] R. Kohavi and G. H. John, "Wrappers for feature subset selection," Artif Intell, vol. 97, no. 1, pp. 273-324, 1997.

[22] H. Kawazoe, et al., "Risk stratification of ventricular fibrillation in Brugada syndrome using noninvasive scoring methods," Heart Rhythm, vol. 13, no. 10, pp. 1947-1954, 2016.

Address for correspondence:

Virginie Le Rolle

LTSI, Universit de Rennes 1

Campus Beaulieau, Bat.22, 35042 Rennes, FRANCE

virginie.lerolle@univ-rennes1.fr 